\title{
Special Section on 2006 International Symposium on Antennas and Propagation
}

The International Symposium on Antennas and Propagation (ISAP) in its series has become one of the most important events in the areas of antennas, wave propagation, and electromagnetic theory, being widely considered as the representative conference in Asia Pacific region in parallel with the IEEE APS International Symposium in USA and also the European Conference on Antennas and Propagation (EuCAP) in Europe. In 2006, the ISAP was successfully held in Singapore on Nov. 1-4, 2006. Over 220 people attended the conference and it attracted strong participation (over 60\%) from Japan. As a continuous support from the IEICE, IEICE Communications Society decided to allocate this special section for the publication of selected papers from this conference in order to share more results and new findings presented at this conference with the larger wireless communications and elecromagnetics communities. We are grateful to IEICE Communications Society for its vision and commitment to this technology.

After the Call-for-Papers for this special section, we have received a total of 79 submissions, including 3 invited papers, 74 regularpapers, and 2 letters. After some hard working by the Editors in the editorial board and many anonymous reviewers who spent a lot of their treasure time to read carefully the manuscripts, we have carefully selected and finally accepted 3 invited papers and 31 regular papers at an acceptance ratio of 34/79 or $43.0 \%$. Generally, the areas of these accepted papers cover (a) antennas, (b) propagation, (c) electromagnetic wave theory, and (d) some other related topics. We certainly hope that you will find the special section helpful in highlighting some of the excellent research results presented in the ISAP'06.

The special section would have not been possible without the significant contributions from a number of professionals. We would take this opportunity to express our sincere appreciation to the authors of this special section for contributing their excellent results to the community, timely revising their manuscript, and adhering strictly to the very tight schedule. Also to reviewers, editorial board members, and IEICE colleagues who helped in the preparation and production of this special section, we are most grateful for your treasure time, great effort, and your professional, dedicated, and invaluable help and/or support.

Special Section Editorial Committee Members

Deputy Editor-in-Chief: Koichi Ito

Guest Editors: Jiro Hirokawa (Tokyo Tech.), Hisato Iwai (Doshisha Univ.), Ryo Yamaguchi (NTT DoCoMo)

Guest Associate Editors: Makoto Ando (Tokyo Tech.), Shuichi Sekine (Toshiba), Jacob Coetzee (National Univ. of Singapore), Zhi Ning Chen (Institute for Infocomm Research), Zhong-Xiang Shen (Nanyang Technological Univ.), Qiang Chen (Tohoku Univ.), Hiroyoshi Yamada (Niigata Univ.), Hisashi Morishita (National Defense Academy), Mitoshi Fujimoto (Univ. of Fukui), Keisuke Noguchi (Kanazawa Inst. Tech.), Kyoichi Iigusa (NICT), Takashi Sekiguchi (Mitsubishi Electric), Toshiyuki Maeyama (KDDI Lab.), Nobuhiro Kuga (Yokohama National Univ.)

\section{Le-Wei Li, Guest Editor-in-Chief}

\begin{abstract}
Le-Wei Li(Member) received his Ph.D. degree in Electrical Engineering from Monash University, Melbourne, Australia, in 1992. Since 1992, he has been with the Dept of Electrical \& Computer Engineering at National University of Singapore where he is currently a Professor and Director of Centre for Microwave and Radio Frequency. His current research interests include electromagnetic theory, computational electromagnetics, radio wave propagation and scattering in various media, microwave propagation and scattering in tropical environment, and analysis and design of various antennas. In these areas, he has (co-)authored 1 book, Spheroidal Wave Functions in Electromagnetic Theory (New York: Wiley, 2001); 45 book chapters, over 260 international refereed journal papers, 31 regional refereed journal papers, and over 260 international conference papers. He is a Fellow of The Electromagnetics Academy (2007-), was IEICE Spore Section Chair (2002-2007), and is a Fellow of IEEE (2005-).

Koichi Ito(Member) received the B.S. and M.S. degrees from Chiba University, Japan, and the D.E. degree from Tokyo Institute of Technology, Japan. He is currently a Professor at the Graduate School of Engineering, Chiba University. He has been appointed as one of the Deputy Vice-Presidents for Research, Chiba University. His main research interests include printed antennas and small antennas for mobile communications, evaluation of the interaction between electromagnetic fields and the human body, and microwave antennas for medical applications. He served as Chair of Technical Group on Human Phantoms for Electromagnetics, IEICE and Chair of the IEEE AP-S Japan Chapter. He currently serves as Vice-Chair of ISAP2008 (Taiwan), General Chair of iWAT2008 (Japan), an Associate Editor for the IEEE Transactions on AP, a Distinguished Lecturer and an AdCom member for the IEEE Antennas and Propagation Society. Professor Ito is a Fellow of the IEEE.
\end{abstract}
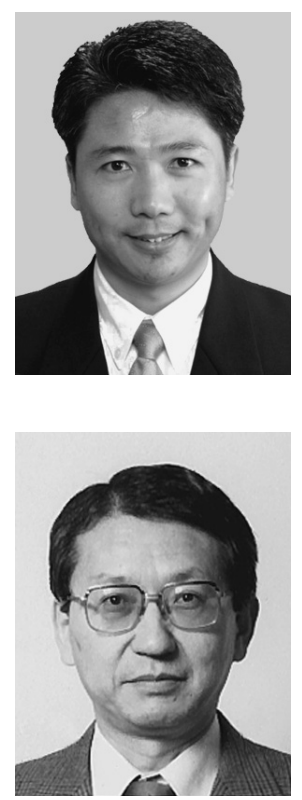